\title{
Tetração e superexponenciais
}

\author{
Marcus Lima
}

\section{Resumo}

Apresentamos uma demonstração de que os valores de $a>0$ para os quais a expressão $a^{a^{a^{*}}}$ está bem definida estão no intervalo $\left[(1 / e)^{e}, e^{1 / e}\right]$. Esse fato foi descoberto e publicado por Euler em 1778 e teve várias demonstrações desde então. Nosso interesse é justificá-lo de modo que tal intervalo surja naturalmente na análise da convergência da expressão acima, usando apenas resultados da primeira disciplina de Cálculo. Nossa abordagem foi baseada nas ideias apresentadas nos artigos da referência.

Palavras-chave: tetração; sequências convergentes; funções exponenciais.

\begin{abstract}
We present a demonstration that the values of $a>0$ for which the expression $a^{a^{a^{*}}}$ is well defined are in the range $\left[(1 / e)^{e}, e^{1 / e}\right]$. This fact was discovered and published by Euler in 1778 and has had several demonstrations ever since. Our interest is to justify this fact so that such an interval arises naturally in the analysis of the convergence of the above expression, using only results from the first discipline of Calculus. Our approach was based on the ideas presented in the reference articles.
\end{abstract}

Keywords: tetration; convergent sequences; exponential functions.

\section{Significado da expressão $a^{a^{a}}$}

Fixado um número $a$, o processo de fazer sucessivas exponenciações com tal número é chamado de tetração, superexponenciação ou ainda hiperexponenciação. Do ponto de vista de uma "ordenação" entre as operações de adição, multiplicação e exponenciação, poderíamos pensar na adição como primeira operação. Considerando a função $F(x)=x+a$ e iniciando com $F(a)$, composições sucessivas de $F$ dão origem à multiplicação (segunda operação). Repetindo o procedimento anterior para $G(x)=a x$, iniciando com $G(a)$, composições sucessivas de $G$ dão origem à exponenciação (terceira operação). Fazendo o mesmo para $H(x)=a^{x}$, iniciando com $H(a)$, composições sucessivas resultam na operação chamada tetração (quarta operação). No entanto, os processos descritos anteriormente são todos finitos. Da mesma forma que ocorrem somatórios e produtórios infinitos, considerando exponenciações infinitas, chega-se à expressão que motiva este texto. 
Como processo infinito, a expressão $a^{a^{a}}$ é definida por meio de um limite. Para cada número real $a>0$ (para não haver restrições quanto à exponenciação), consideramos a sequência $\left(a_{n}\right)_{n=1}^{\infty}($ que daqui em diante denotaremos apenas por $\left.\left(a_{n}\right)\right)$ definida por:

$$
a_{1}=a \quad \text { e } a_{n+1}=a^{a_{n}} \quad \text { para } \quad n \geq 1 .
$$

Definimos

$$
a^{a^{a^{\prime}}}=\lim _{n \rightarrow \infty} a_{n}
$$

se esse limite existir.

Para $a=1,\left(a_{n}\right)$ é convergente, com $\lim _{n \rightarrow \infty} a_{n}=1$. Para $a \neq 1$, se $\left(a_{n}\right)$ é convergente, digamos $\lim _{n \rightarrow \infty} a_{n}=x$, então

$$
x=a^{a^{a^{\prime}}}=\lim _{n \rightarrow \infty} a_{n}=\lim _{n \rightarrow \infty} a^{a_{n-1}}=a^{\lim _{n \rightarrow \infty} a_{n-1}}=a^{x}
$$

em que a última igualdade decorre da continuidade da função exponencial $a^{x}$.

Assim, um número real a para o qual a sequência $\left(a_{n}\right)$ é convergente, com $\lim _{n \rightarrow \infty} a_{n}=x$, satisfaz a equação $x=a^{x}$ ou $a=x^{1 / x}$, que nos leva ao estudo da função $f(x)=x^{1 / x \rightarrow \infty}, x>0$. Tal função assume valor máximo $e^{1 / e}$ quando $x=e\left(f^{\prime}(x)=x^{1 / x} \frac{1}{x^{2}}(1-\ln x)>0\right.$, para $x<e$ e $f^{\prime}(x)<0$, para $x>e$ ) e tem o gráfico:

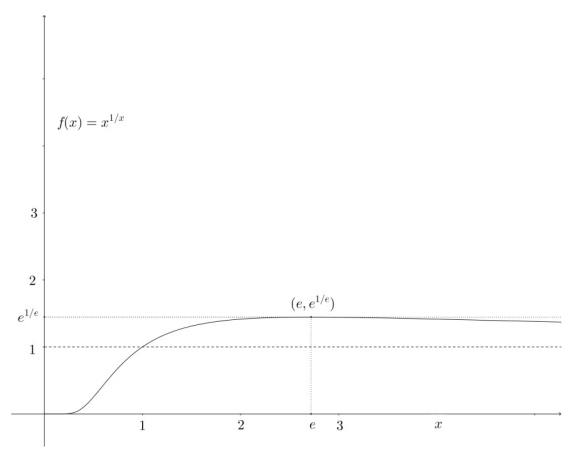

Figura 1: Gráfico da função $f(x)=x^{1 / x}$

Disso decorre que uma condição necessária para $\left(a_{n}\right)$ ser convergente é $a \leq e^{1 / e}$. Isso determina o limite superior do intervalo de convergência de $\left(a_{n}\right)$ e e como valor máximo para o limite de $\left(a_{n}\right)$. Com isso restringiremos a análise das sequências $\left(a_{n}\right)$ para $0<a \leq e^{1 / e}$. Como para $a=1$, $\lim _{n \rightarrow \infty} a_{n}=1$, analisaremos separadamente os intervalos $\left(1, e^{1 / e}\right]$ e $(0,1)$.

\section{Convergêngia no intervalo $\left(1, e^{1 / e}\right]$}

Com $a>1$, iterando as exponenciais, vem $a<a^{a}<a^{a^{a}}<\ldots$, e a sequência $\left(a_{n}\right)$ é crescente. Também, $a_{1}=a \leq e^{1 / e}$, assim, $a_{2}=a^{a_{1}} \leq\left(e^{1 / e}\right)^{e^{1 / e}}=e^{e^{1 / e-1}} \leq e$. Por indução finita podemos mostrar que $a_{n} \leq e$, para todo $n$. Ou seja, para cada número real $a$ satisfazendo $1<a \leq e^{1 / e}$, a sequência $\left(a_{n}\right)_{n=1}^{\infty}$ é crescente e limitada superiormente, portanto é convergente. 


\section{Análise de convergência no intervalo $(0,1)$}

Neste caso $a_{n}<1$, para todo $n$. Além disso, $a=a_{1}<a_{2}=a^{a_{1}}<1$. Analogamente, $a^{a}=a_{2}>$ $a_{3}=a^{a^{a}}<1$ e $a=a_{1}<a_{3}=a^{a^{a}}$. Da última desigualdade, decorre que $a^{a_{1}}=a_{2}>a_{4}=a^{a_{3}}$. Novamente por indução finita sobre $n$, obtemos $a=a_{1}<a_{3}<\cdots<a_{2 n+1}$ e $a_{2 n}<\cdots<a_{4}<a_{2}=$ $a^{a}$ para todo $n$ natural. Assim, $\left(a_{2 n+1}\right)$ é crescente e limitada superiormente e $a_{2 n}$ é decrescente e limitada inferiormente, logo ambas são convergentes com limites $A$ e $B$, respectivamente, e satisfazem: $A=\lim a_{2 n+1}=\lim a^{a^{a_{2 n-1}}}=a^{a^{A}}$ e $B=\lim a_{2 n}=\lim a^{a^{a_{2(n-1)}}}=a^{a^{B}}$. Nosso interesse agora é determinar os valores de $a$ para os quais teremos $A=B$ ou, equivalentemente, determinar os valores de $a$ de modo que a equação $a^{a^{x}}=x$ tenha solução para um único valor de $x \geq 0$. Faremos isso com base no estudo da função $\varphi_{a}(x)=a^{a^{x}}, x \geq 0$, procurando condições sobre $a$ para que o gráfico de $\varphi_{a}(x)$ corte a reta $y=x$ em apenas um ponto (ou seja, para que $\varphi_{a}(x)$ tenha uma única solução). Fixemos a tal que $0<a<1$. Temos $\varphi_{a}^{\prime}(x)=a^{a^{x}} a^{x}(\ln a)^{2}>0$, para todo $x \geq 0$, portanto, $\varphi_{a}(x)$ é crescente. Além disso, a reta $y=1$ é uma assíntota horizontal ao gráfico $\operatorname{de} \varphi_{a}(x)\left(\lim _{x \rightarrow \infty} a^{a^{x}}=1\right.$, pois $\left.0<a<1\right)$.

Se $\lim _{n \rightarrow \infty} a_{2 n+1}=B$ e $\lim _{n \rightarrow \infty} a_{2 n}=B$, usando as igualdades em (1), obtemos $\lim _{n \rightarrow \infty} a_{n}=B$, portanto, $a^{B}=\stackrel{n \rightarrow \infty}{=}$.

Observemos que $B$ é uma solução de $a^{a^{x}}=x$. Usando isso e reescrevendo $\varphi_{a}^{\prime}(x) a^{a^{x}} \ln a^{a^{x}} \ln a$, temos que $\varphi_{a}^{\prime}(B)=a^{a^{B}} a^{B}(\ln a)^{2}=B \ln B \ln a=(\ln B)^{2}$. Agora, se $\varphi_{a}^{\prime}(B)>0$, no ponto $\left(B, \varphi_{a}(B)\right)$, em que o gráfico de $\varphi_{a}(x)$ corta a reta $y=x$, a inclinação da reta tangente ao gráfico de $\varphi_{a}(x)$ é maior do que 1. No entanto, como $y=1$ é assíntota horizontal, deve haver outro cruzamento desse gráfico com a reta $y=x$, ou seja, haverá mais de uma solução da equação $a^{a^{x}}=x$. Disso concluímos que se há uma única solução $B$ de $a^{a^{x}}=x$, então $\varphi_{a}^{\prime}(B) \leq 1$. Isso nos remete a encontrar todos os valores de $a$ para os quais $\varphi_{a}^{\prime}(x) \leq 1$, para todo $x \geq 0$. Para isso vamos investigar um pouco mais a função $\varphi_{a}^{\prime}(x)$. Calculando sua derivada, obtemos $\varphi_{a}^{\prime \prime}(x)=a^{a^{x}} a^{x}(\ln a)^{3}\left(a^{x} \ln a+1\right)$. Resolvendo $\varphi_{a}^{\prime \prime}(x)=0$ (que ocorre para $a^{x} \ln a+1=0$ ), obtemos $a^{a^{x}}=e^{-1}$. Como $\varphi_{a}^{\prime \prime}(x)>0$ para todo $x \geq 0$ tal que $a^{a^{x}}<e^{-1}$ e $\varphi_{a}^{\prime \prime}(x)<0$ para todo $x \geq 0$ tal que $a^{a^{x}}>e^{-1}$, concluímos que:

i. o máximo de $\varphi_{a}^{\prime}(x)$ ocorre quando $a^{a^{x}}=e^{-1}$.

ii. o gráfico de $\varphi_{a}(x)$ tem um ponto de inflexão cuja abcissa é $x_{a}=\frac{1}{\ln a} \ln \left(-\frac{1}{\ln a}\right) \geq 0$, que é o ponto de máximo de $\varphi_{a}^{\prime}(x)$. A expressão para $x_{a}$ é obtida resolvendo $a^{x} \ln a+1=0$. Da condição $x_{a} \geq 0$, decorre $0<a \leq e^{-1}$.

Como consequência de i. acima, $\varphi_{a}^{\prime}(x)=a^{a^{x}} a^{x}(\ln a)^{2}=a^{a^{x}} \ln a^{a^{x}} \ln a \leq-\frac{1}{e} \ln a$, para todo $x \geq 0$. Assim, $\varphi_{a}^{\prime}(x) \leq 1$ para todo $x \geq 0$ quando $-\frac{1}{e} \ln a \leq 1$, ou seja, $a \geq e^{-e}$. Vamos mostrar que, para todo $a$ no intervalo $\left[e^{-e}, 1\right)$, o gráfico de $\varphi_{a}(x)$ corta a reta $y=x$ apenas uma vez. Observemos que isso implica que $\lim _{n \rightarrow \infty} a_{2 n+1}=\lim _{n \rightarrow \infty} a_{2 n}$ e, portanto, a existência $\lim _{n \rightarrow \infty} a_{n}$. Nossa análise será baseada em ii. acima. Para $a=e^{-e}, I=\left(\frac{1}{e}, \frac{1}{e}\right)$ é o ponto de inflexão do gráfico de $\varphi_{a}(x)$ e o gráfico de $\varphi_{e^{-e}}(x)$ corta a reta $y=x$ apenas uma vez, no ponto $I$. Para $e^{-e}<a<e^{-1}$, como $\varphi_{a}^{\prime}(x)<1$ para todo $x \geq 0, \varphi_{a}(0)=a>0, \varphi_{a}(x)$ é crescente e $\lim _{x \rightarrow \infty} \varphi_{a}(x)=1$, o gráfico de $\varphi_{a}(x)$, $x \geq 0$, corta a reta $y=x$ em um único ponto. Para $e^{-1} \leq a<1$, o gráfico de $\varphi_{a}(x), x \geq 0$, tem concavidade para baixo e corta a reta $y=x$ em um único ponto. Em particular, $x=0$ é a abcissa do ponto de inflexão do gráfico de $\varphi_{e^{-1}}(x)$.

Finalmente, para $a$ no intervalo $\left(0, e^{-e}\right), \lim _{n \rightarrow \infty} a_{2 n+1}=B>a$ pois $\left(a_{2 n+1}\right)$ é crescente. Além disso, 


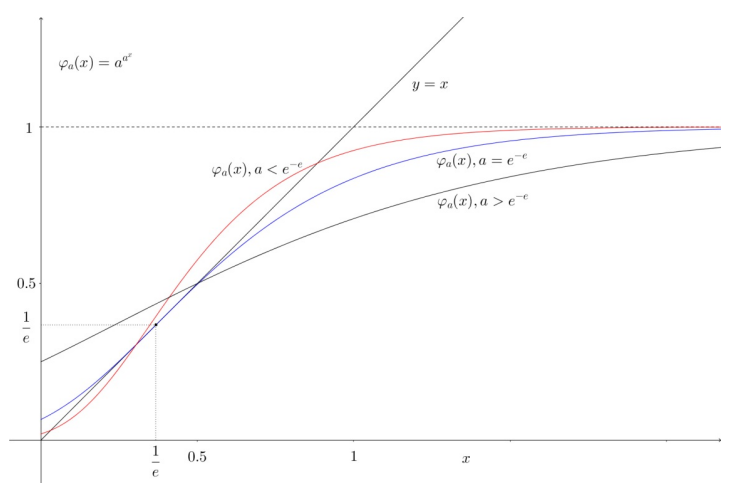

Figura 2: Gráficos de $\varphi_{a}(x)$ para $a<e^{-e}$ (em vermelho); $a=e^{-e}$ (em azul) e $e^{-e}<a<1$ (em preto)

$\varphi_{a}(B)=B$ e $\varphi_{a}^{\prime}(B)=a^{a^{B}} a^{B}(\ln a)^{2}=B \ln B \ln a>a \ln a \ln a>(\ln a)^{2}>e^{2}>1$, em que na segunda desigualdade acima usamos que $a^{a}>a$. Como discutido anteriormente, a desigualdade acima implica a existência de uma outra solução de $\varphi_{a}(x)=x$ e podemos concluir que não existe $\lim _{n \rightarrow \infty} a_{n}$. Dessa análise da função $\varphi_{a}(x)=a^{a^{x}}, x \geq 0$, podemos concluir que, para $e^{-e} \leq a<1$, a equação $a^{a^{x}}=x$ tem apenas uma solução para $x \geq 0$. Dessa forma, a expressão $a^{a^{a^{\prime}}}$ está bem definida (no sentido que para todo $a$ a expressão assume apenas um valor) para $a$ no intervalo $\left[(1 / e)^{e}, e^{1 / e}\right]$.

\section{Referências}

[1] Creutz, M.; Sternheimer, R. M. On the convergence of iterated exponentiation - II. Fibonacci Quarterly v. 19, pp326-336, 1981.

[2] Knoebel, R. A. Exponentials reiterated. Amer. Math. Monthly v. 88, pp235-252, 1981.

[3] Rippon, P. J. Infinite exponentials. The Math. Gazzete, v. 67, n. 441, pp189-196, 1983.

Marcus Lima Departamento de Matemática-UFSCar <lima@dm.ufscar.br>

Recebido: 2016

Publicado: 2016 